\title{
Lamination parameters for sandwich and hybrid material composites
}

\author{
Gustavo H. C. Silva* and Yasser Meddaikar ${ }^{\dagger}$ \\ DLR - German Aerospace Center, Göttingen, 37073, Germany
}

\section{Nomenclature}

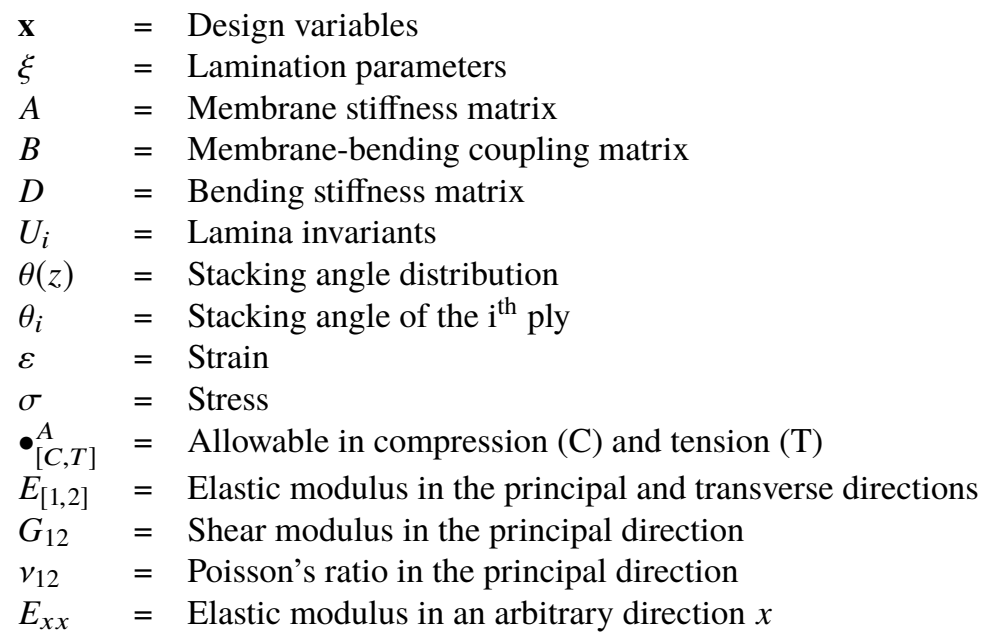

\section{Introduction}

$\Gamma^{H E}$ development of lamination parameters [1,2] allowed for a revolution in the optimization methods of laminated composites. Lamination parameters provide a convenient way to bypass discrete stacking sequence definitions and of iterating through stiffness components of a laminate in a continuous and convex design space. Together with advances in lamination parameter constraints [3-5] which forces the design space to be consistent with a number of stacking sequence design criteria, large-scale gradient-based optimizers have allowed for composites to be optimized to meet an increasing number of complex multidisciplinary requirements. Ever since, researchers have used lamination parameters in increasingly sophisticated aerospace applications [6-12]; the majority of them focusing on composites consisting of a single material basis.

In this paper, the lamination parameters scheme is extended to represent multi-material layups. The scheme will hopefully enable similar optimization methods to include mixed-material configurations, sandwich structures, mixed textile and unidirectional layups and fibre metal laminates (FMLs) such as Aramid Reinforced Aluminum Laminate (ARALL), Glass Reinforced Aluminum Laminate (GLARE) and Carbon Reinforced Aluminum Laminate (CARALL). This extension will help to cover an important range of current and future applications in the composites industry. The present work is applicable to optimization cases where the different material groups and their order are pre-defined.

Sandwich composite optimization for a wide range of applications has been presented [13-20]. Among these, very few approaches using lamination parameters are available, which is the focus of this work. Miki et al. [13] define a modified set of lamination parameters specific to sandwich composites, using the approach to make graphical studies on the Miki-diagram. Balabanov et al. [17] optimize a sandwich panel with in-plane loading using existing lamination parameters together with approximations for the bending stiffness matrix. Weckner et al. [18] improve on the work, proposing a generalized scheme without limitation on the number of material systems or material properties. Jin et al. [19] apply lamination parameters to the design composite sandwich panels with aeroelastic constraints, using an

\footnotetext{
* Research Scientist at DLR Institute of Aeroelasticity

${ }^{\dagger}$ Research Scientist at DLR Institute of Aeroelasticity
} 
approximated bending stiffness matrix. Such approximations for the bending stiffness matrix work well for sandwich composites with thick cores and thin facesheets.

These approaches generally make strong assumptions on the structures they aim to model, such as for thin facesheets and thick core combinations, leading either to inaccuracies in the stiffness calculations, loss of continuity in the transition between standard laminates and those with multiple materials, or have a significantly different form from classical lamination parameters [6]. The formulation presented in this paper addresses these issues with respect to optimizing multimaterial composites using lamination parameters. A less restrictive set of assumptions is proposed. Through its simplicity, the proposed scheme will allow direct application of the available lamination parameter bounds for compatibility of angle-types, manufacturing, and blending with little or no modifications at all. design.

The paper begins by discussing the parametrization scheme and concludes with an application to sandwich panel

\section{Stiffness Calculation}

A typical single-material stacking sequence is shown in Figure 1. The stiffness matrices for this well-known configuration will be used as the basis for the creation of the hybrid material cases. To review the standard notation, taking $\bar{Q}_{i}$ as the rotated ply stiffness matrix, and $z_{i}$ as the coordinate of the $\mathrm{i}^{\text {th }}$ ply face, the effective stiffness of such an arrangement is obtained from the classical definitions of the matrices for membrane (A), coupling (B) and bending stiffness (D),

$$
\begin{aligned}
{[A, B, D] } & =\left[1, \frac{1}{2}, \frac{1}{3}\right] \sum_{i=1}^{n} \bar{Q}_{i}\left[\left(z_{i}-z_{i-1}\right),\left(z_{i}^{2}-z_{i-1}^{2}\right),\left(z_{i}^{3}-z_{i-1}^{3}\right)\right] \\
& =\left[1, \frac{1}{2}, \frac{1}{3}\right]\left(\sum_{i=1}^{n / 2} \bar{Q}_{i}[\cdots]+\sum_{i=n / 2+1}^{n} \bar{Q}_{i}[\cdots]\right) \\
& =\left[A_{u}, B_{u}, D_{u}\right]+\left[A_{l}, B_{l}, D_{l}\right] .
\end{aligned}
$$

Here, their definitions are split into the summations for the upper and lower halves of the stack, indicated by the subscripts $u$ and 1 respectively; this division will be useful momentarily. For symmetric laminates, the upper and lower parts of the A and D matrices are equal, $A_{u}=A_{l}$ and $D_{u}=D_{l}$, while for the coupling matrix B they are negatives of each other $B_{u}=-B_{l}$.

\section{A. Offset Laminates}

The effective stiffness of hybrid material layups can be calculated by offsetting and superposing the layers of each material type as the example shown in Figure 3. In this example the core material is at the center and the unidirectional lay-ups are split into two and are offset a distance of $h / 2$ at each side.

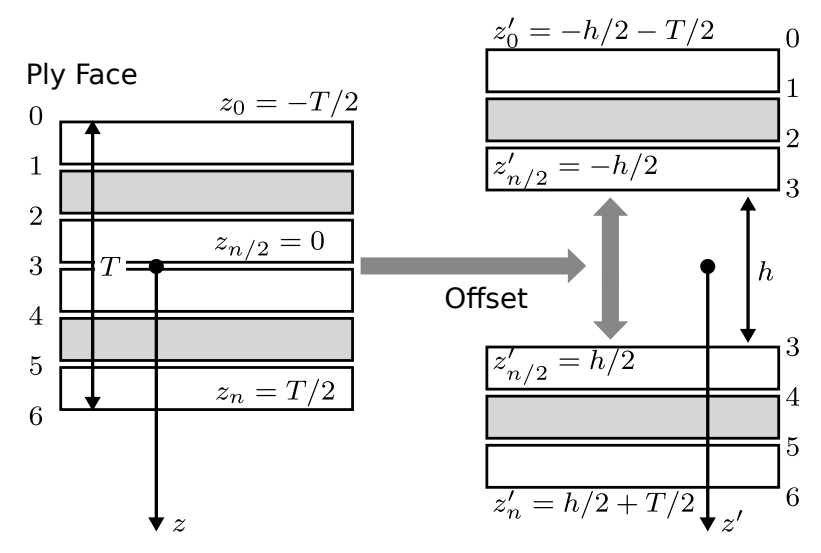

Fig. 1 Coordinate system for standard laminates and for ones rigidly offset by a distance $h$ 
For this purpose, imagine a conventional laminated composite with an even number of plies as shown in Figure 1. The stack is divided in two, split apart by a distance $h$ and held separated by an ideal material infinitely rigid in the $z$-direction, but with finite stiffness in other directions. The usefulness of this is that a mixed materials structure such as a sandwich-core composite can be produced by superposing offset laminates. The stiffness of the resulting laminate can be determined by offsetting the positive $z_{i}$ ply coordinates by $h / 2$ and the negative ones by $-h / 2$,

$$
z_{i}^{\prime}=\left\{\begin{array}{lll}
z_{i}-h / 2 & \text { for } & i \in[0, \cdots, n / 2] \\
z_{i}+h / 2 & & i \in[n / 2, \cdots, n]
\end{array}\right.
$$

Substituting the offset values $z_{i}^{\prime}$ into the A, B, D summations yields the offset matrices.

$$
\begin{gathered}
A^{\prime}=A \\
B_{u}^{\prime}=B_{u}-\frac{h}{2} A_{u} \quad \& \quad B_{l}^{\prime}=B_{l}+\frac{h}{2} A_{l} \\
B^{\prime}=B_{u}^{\prime}+B_{l}^{\prime}= \begin{cases}B+\frac{h}{2}\left(A_{l}-A_{u}\right) & \text { general laminates } \\
0 & \text { symmetric }\end{cases} \\
D^{\prime}= \begin{cases}D+h\left(B_{l}-B_{u}\right)+\frac{1}{4} h^{2} A & \text { general laminates } \\
D-2 h B_{u}+\frac{1}{4} h^{2} A & \text { symmetric }\end{cases}
\end{gathered}
$$

\section{B. Stiffness Superposition}

Using the offset formulas, the stiffness of hybrid material composites can now be constructed by combinations of the A, B, D matrices of multiple layer blocks. Figure 2 introduces the notation used hereafter.

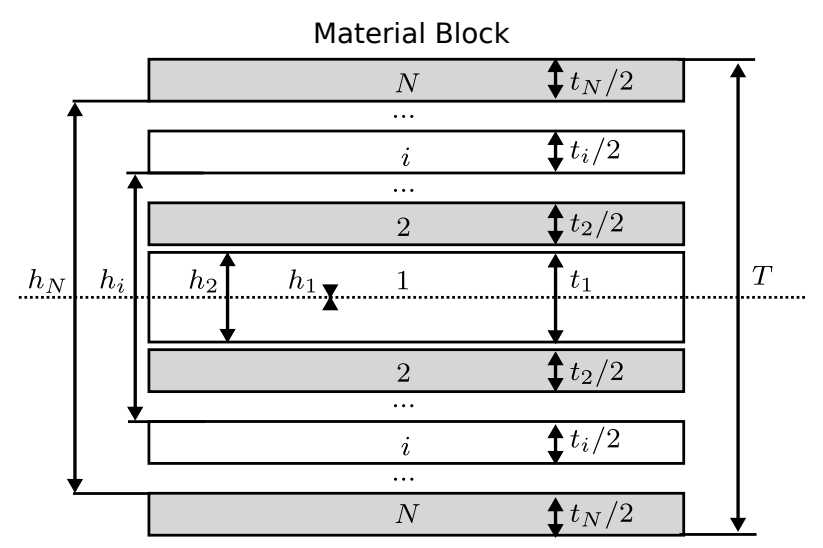

Fig. 2 Notation for a hybrid material layup using offset material blocks

For an N-block material, assuming that each material block has an even number of plies with thickness $t_{k}$, and is split in half. Starting with the innermost block, the effective offset height of each block is

$$
h_{i}= \begin{cases}0 & \text { for } i=1 \\ \sum_{k=1}^{i-1} t_{k} & \text { for } i>0\end{cases}
$$

The stiffness matrices for general layups is

$$
A=\sum_{i=1}^{N} A_{i}
$$




$$
\begin{gathered}
B_{u}=\sum_{i=1}^{N} B_{u, i}-\frac{h_{i}}{2} A_{u, i}, \quad B_{l}=\sum_{i=1}^{N} B_{l, i}+\frac{h_{i}}{2} A_{l, i}, \\
D= \begin{cases}\sum_{i=1}^{N} D_{i}+h_{i}\left(B_{l, i}-B_{u, i}\right)+\frac{h_{i}^{2}}{4} A_{i} & \text { general laminates } \\
\sum_{i=1}^{N} D_{i}-2 h_{i} B_{u, i}+\frac{h_{i}^{2}}{4} A_{i} & \text { symmetric }\end{cases}
\end{gathered}
$$

\section{Lamination Parameters}

Lamination parameters for these matrices are defined as

$$
\begin{aligned}
& \xi_{[1,2,3,4]}^{A}=\frac{1}{2} \int_{-1}^{1}[\cos 2 \theta(\bar{z}), \cos 4 \theta(\bar{z}), \sin 2 \theta(\bar{z}), \sin 4 \theta(\bar{z})] d \bar{z} \\
& \xi_{[1,2,3,4]}^{B u}=-2 \int_{-1}^{0}[\cos 2 \theta(\bar{z}), \cos 4 \theta(\bar{z}), \sin 2 \theta(\bar{z}), \sin 4 \theta(\bar{z})] \bar{z} d \bar{z} \\
& \xi_{[1,2,3,4]}^{B l}=2 \int_{0}^{1}[\cos 2 \theta(\bar{z}), \cos 4 \theta(\bar{z}), \sin 2 \theta(\bar{z}), \sin 4 \theta(\bar{z})] \bar{z} d \bar{z} \\
& \xi_{[1,2,3,4]}^{D}=\frac{3}{2} \int_{-1}^{1}[\cos 2 \theta(\bar{z}), \cos 4 \theta(\bar{z}), \sin 2 \theta(\bar{z}), \sin 4 \theta(\bar{z})] \bar{z}^{2} d \bar{z}
\end{aligned}
$$

where $\bar{z}$ is the normalized fiber distance $\bar{z}=z / T$ and $\theta(\bar{z})$ is the stacking angle distribution. The lamination parameters for matrix $B$ are $2 \xi^{B}=\xi^{B l}-\xi^{B u}$. Each of the components of the bending and stiffness matrices can be obtained by defining a lamination parameter matrix and vector of invariants,

$$
\begin{gathered}
{[\xi]=\left[\begin{array}{ccccc}
1 & \xi_{1} & \xi_{2} & 0 & 0 \\
1 & -\xi_{1} & \xi_{2} & 0 & 0 \\
0 & 0 & -\xi_{2} & 1 & 0 \\
0 & 0 & -\xi_{2} & 0 & 1 \\
0 & \xi_{3} / 2 & \xi_{4} & 0 & 0 \\
0 & \xi_{3} / 2 & -\xi_{4} & 0 & 0
\end{array}\right],} \\
\left\{\begin{array}{l}
U_{1} \\
U_{2} \\
U_{3} \\
U_{4} \\
U_{5}
\end{array}\right\}=\frac{1}{8}\left[\begin{array}{rrrr}
3 & 3 & 2 & 4 \\
4 & -4 & 0 & 0 \\
1 & 1 & -2 & -4 \\
1 & 1 & 6 & -4 \\
1 & 1 & -2 & 4
\end{array}\right]\left\{\begin{array}{l}
Q_{11} \\
Q_{22} \\
Q_{12} \\
Q_{66}
\end{array}\right\} .
\end{gathered}
$$

A vector of components for each of the stiffness matrices can be obtained by a simple matrix vector multiplication,

$$
\begin{aligned}
& \left\{\hat{A}, \hat{B}_{u}, \hat{B}_{l}, \hat{D}\right\}=\left[\xi^{A}, \xi^{B u}, \xi^{B l}, \xi^{D}\right]\{U\}
\end{aligned}
$$

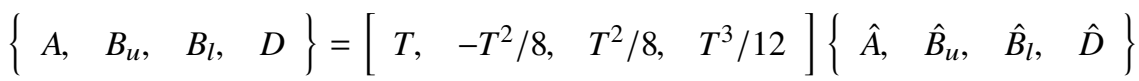

where the order of the components of each of these vectors is

$$
\{\bullet\}=\left\{\begin{array}{llllll}
\bullet_{11} & \bullet_{22} & \bullet_{12} & \bullet_{66} & \bullet_{16} & \bullet_{26}
\end{array}\right\}
$$

The coefficients in the lamination parameter definition in Equation (12)-(13) are selected to satisfy the same trigonometric identities as other lamination parameters [21]. 


\section{Results}

Advances in material sciences and in applied optimization methodology have led to an interesting phenomenon in aerospace structures: designs appear to be ever-more stiffness dominated than strength dominated. In the wing of a typical transport aircraft for instances, we see that most skin panels are sized by buckling stability and handling requirements rather than strength; with as much as one-third of panels (those in the outboard regions) sized by minimum gauge $[12,22,23]$. Development of the next generation of materials will likely deliver a further increase in the strength-to-weight ratio of panels, with carbon nanotube technology for instance, having a potential order-of-magnitude improvement in both stiffness and strength when compared to IM7.

As a consequence of these advances, one can expect that panels designed in traditional methods will easily satisfy strength requirements but will be too thin to prevent buckling. At present, sandwich panels are an engineering favorite for exactly this situation. Rather than increasing the thickness of the facesheets, there is an expected weight benefit in increasing the bending stiffness by adding an ultra-light core instead. The problem with sandwiches however, is that adding a core material will subject the panel to a large set of new and often complex failure mechanisms; many of which are unfamiliar to the workflow of a complex structural design and are often too involved for a procedural design approach. In order to seriously consider a sandwich panel concept, the design method must account for these failure mechanisms as well as satisfy a number of challenging practical concerns [24, 25].

In the rest of this section, with the aid of the lamination parameters scheme developed in this paper, the design-space of a family of sandwich panels will be explored and analyzed. The exploration begins by discussing the potential pitfalls of available stiffness approximation schemes and concludes by producing design curves for a panel under compression loading.

\section{A. Geometry, Boundary Conditions and Failure Modes}

Figure 3 shows a schematic of a material arrangement common in aerospace applications. This sandwich composite consists of a lightweight core, usually foam or Aramid honeycomb, and a stacking sequence of unidirectional carbon fiber layers providing stiffness and strength. Sometimes a single layer of covering material, usually carbon fiber or fiberglass for damage and handling tolerance is also used.



Fig. 3 Compression of a sandwich composite

In the example here, the facesheets are made of Hexcel IM7 5052 carbon-epoxy laminates with a ply thickness of $0.128 \mathrm{~mm}$ and the core material is Hexcel Aramid honeycomb HRH10. Table 1 summarizes their material properties.

The strain limits for laminated facesheets are generally dependent on directional stiffness. The maximum load which results in each of the failure mechanisms shown in Figure 3 are dependent on the membrane and bending stiffness matrices, and will be described hereafter.

Facesheet failure will be determined in this paper using a common technique employed in industry known as the Angle Minus Longitudinal (AML) method [26]. AML refers to the percentage of $\pm 45^{\circ}$ plies (angle) minus the percentage of plies at $0^{\circ}$ (longitudinal), a metric for which laminates of similar values tend to possess the same failure 
Table 1 Material Properties

\begin{tabular}{ccccc}
\hline \hline Density $[\mathrm{kg} / \mathrm{m} 3]$ & \multicolumn{4}{c}{ Property } \\
\hline \multicolumn{5}{c}{ Carbon-Epoxy Facesheets: Hexcel-8552 UD IM7 } \\
\multirow{4}{*}{1580} & $E_{1}$ & $E_{2}$ & $G_{12}$ & $v_{12}$ \\
& $160 \mathrm{GPa}$ & $20 \mathrm{GPa}$ & $10 \mathrm{GPa}$ & 0.27 \\
& Honeycomb Core (3mm Cell): Hexcel-HRH10 & \\
64 & $E_{1}$ & $E_{z}$ & $G_{1 z}$ & $G_{2 z}$ \\
64 & $<0.001 \mathrm{GPa}$ & $0.19 \mathrm{GPa}$ & $0.063 \mathrm{GPa}$ & $0.035 \mathrm{GPa}$ \\
\hline \hline
\end{tabular}

modes in notched or damaged compression tests. Open-hole compression tests (OHC), as shown in Figure 4 can then be used as a direct laminate-level empirical strain allowable. For this criteria, only the facesheet thickness and its layup angles needs to be known.

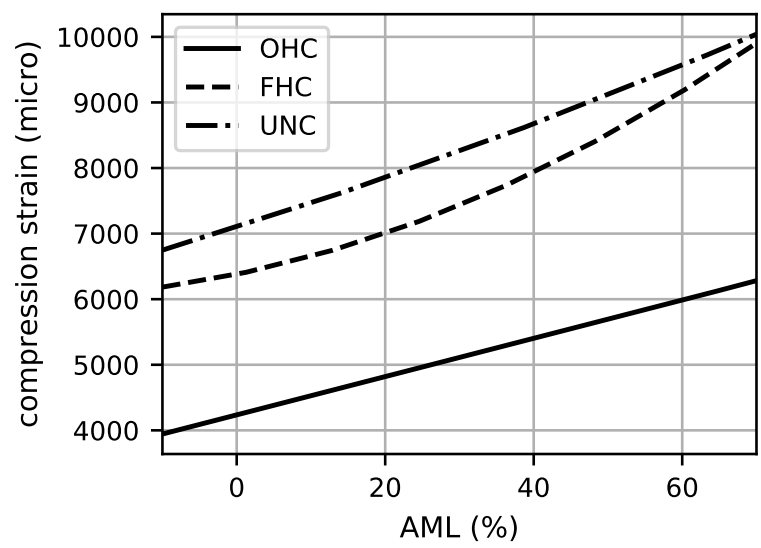

Fig. 4 Variation of stiffness and allowable strains with AML for a carbon-reinforced IM7 composite - NIAR $[26,27]$ (OHC - open hole compression, FHC - filled hole compression, UNC - unnotched compression)

For a flat rectangular sandwich column, the critical compressive force per unit width on the facesheets can calculated by an expression found in $\mathrm{CMH}-17$ [28],

$$
N_{b k}=\frac{(\pi / \beta L)^{2} D_{x x}}{1+(\pi / \beta L)^{2}\left(D_{x x} / S\right)}
$$

where $L$ is the length of the column, $D$ is the bending stiffness, $S$ is the effective transverse shear stiffness and $\beta$ depends on the boundary condition such that $\beta L$ is the effective plate length. $\beta=0.5$ is taken for the examples in this paper representing a clamped support on both ends of the column. The presence of a sandwich core in the laminate makes the effects of tranverse shear important to consider. If not accounted for, the buckling load can be quite conservative when compared to traditional Euler-buckling calculations. The transverse shear stiffness is dependent on the thickness of the core and facesheet and the transverse shear stiffness of the core and is represented as in the CMH-17 [28] by,

$$
S=\frac{G_{1 z}\left(t_{c}+t_{f} / 2\right)^{2}}{t_{c}}
$$

Alternatively, theories such as a first or higher order shear deformation theory [29] can be used to include the transverse shear stiffness.

Crimping is the final failure mode to be investigated here. This failure mode is a local instability which occurs when the shear stiffness of the core is too low compared to the facesheet stiffness. A simple expression for crimping load can be obtained from CMH-17 (Eq. 4.6.7(a)), 


$$
N_{c r}=\gamma \frac{\left(t_{c}+t_{f} / 2\right)^{2}}{t_{c}} G_{c}
$$

where $\gamma$ is a knockdown factor, set to 0.95 .

Note that the buckling load depends on the D matrix of the sandwich, which in turn depends on $A_{f}, D_{f}, B_{u, f}$ and $t_{c}$. The OHC load depends only on $A_{f}$, while the crimping load depends on $t_{f}, t_{c}$ and $G_{c}$.

In the following studies, a column length of $L=1 \mathrm{~m}$, equivalent length factor $\beta=0.5$, material properties in Table 1 , limits for core thickness $1 \mathrm{~mm} \leq t_{c} \leq 50 \mathrm{~mm}$ and limits for facesheet thickness $0.5 \mathrm{~mm} \leq t_{f} \leq 12.8 \mathrm{~mm}$ (corresponding to number of plies between 4 and 100 with a single ply thickness of $0.128 \mathrm{~mm}$ ) are considered.

\section{B. Bending Stiffness Effects}

Considering symmetric layups only, the stiffness matrices of the sandwich is

$$
\begin{aligned}
& A=A_{c}+A_{f} \\
& B=0 \\
& D=D_{c}+D_{f}-2 t_{c} B_{u, f}+\frac{1}{4} t_{c}^{2} A_{f}
\end{aligned}
$$

It is common in sandwich optimization to approximate the previous equations with simpler versions which neglect the $B_{u}$ matrix all together. Equations such as the ones in $[17,19,30]$ are commonly employed in optimization,

$$
D \approx D_{c}+D_{f}+A_{f}\left(\frac{t_{c}}{2}+\frac{t_{f}}{4}\right)^{2}
$$

This approximation is tempting because it avoids the unfamiliar $B_{u}$ matrix and reduces by four the number of optimization parameters.

The effects of this approximation is not always acceptable, as is shown in the Monte Carlo analysis in Figure 5. Here 2000 sandwich panels were constructed by drawing random number of facesheet plies at $0^{\circ}, \pm 45^{\circ}$ and $90^{\circ}$, between 4 and 100 plies, and a random core thickness between $1 \mathrm{~mm}$ and $50 \mathrm{~mm}$. The buckling loads are normalized by the facesheet's $\mathrm{OHC}$ equivalent load, which allows analyzing a wide range of lightly to heavily loaded panels. The error (\%) in Figure 5 is calculated with respect to the buckling loads obtained using Equation 22, considered here to be the exact solution. The Monte Carlo simulation shows that using Equation 23 for the $D$ stiffness matrix, which neglects the $B_{u}$ matrix can lead to important errors in the calculation of the buckling load factor, nearing $20 \%$ for the considered sandwich column. Furthermore it shows the approximation is typically non-conservative.

A better approximation of the D matrix can be developed by taking advantage of the structure of the lamination parameters in equations (11)-(15). Since the lamination parameters $\xi^{A}, \xi^{B u}$, and $\xi^{D}$ are zeroth, first and second moments of the same angle distribution, it is not unreasonable to assume that the values parameters $\xi^{B u}$ are somewhere in between $\xi^{A}, \xi^{D}$. Figure 6 shows the strong linear correlation between the components.

Taking a simple average, $\xi^{B u}=1 / 2\left(\xi^{A}+\xi^{D}\right)$, yields the following approximation,

$$
D \approx D_{c}+\left(1+\frac{3 t_{c}}{2 t_{f}}\right) D_{f}+\left(\frac{t_{c} t_{f}}{8}+\frac{1}{4} t_{c}^{2}\right) A_{f}
$$

Using the approximation in Equation 24 for the bending stiffness matrix $D$ indeed leads to results with a much lower error in the buckling loads in the order of $5 \%$, as seen in Figure 5.

\section{Panel Design Curves}

In this section design curves are presented showing a comparison of the maximum load bearing capacity of sandwich panels versus standard single-material layups. The maximum load a panel can withstand can be determined by solving the following optimization problem, where $p$ is the panel load, 

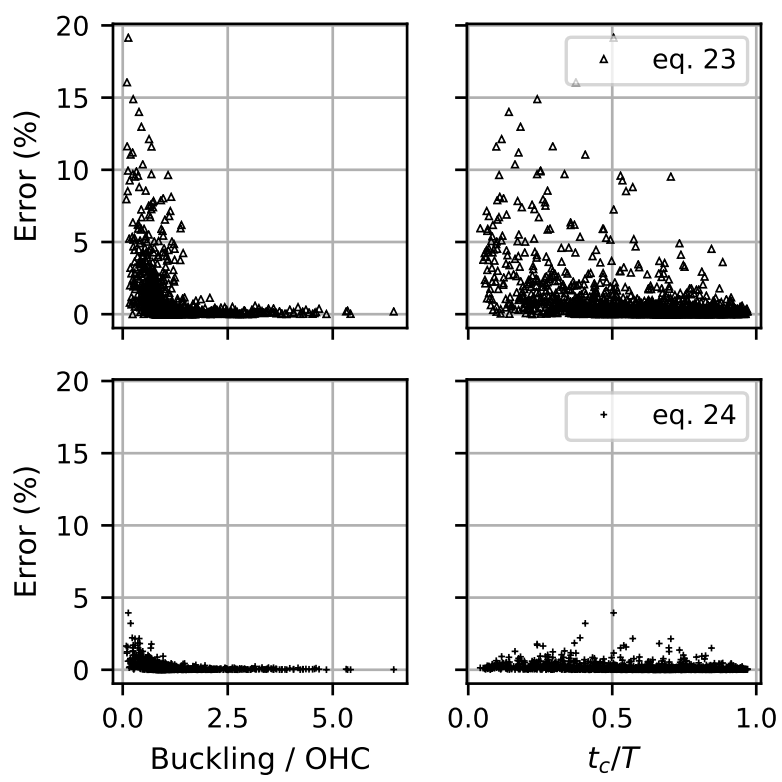

Fig. 5 Error in buckling load factor for different approximations of the bending stiffness $D$

$$
\begin{array}{rll}
\max _{p, \mathbf{x}} & p & \\
\text { subject to: } & P_{b k}(\mathbf{x}) \geq p & \text { Buckling } \\
& P_{c r}(\mathbf{x}) \geq p & \text { Crimping } \\
& C_{k}(\mathbf{x}) \geq 1 & \text { Admissibility } \\
& x_{i}^{l} \leq x_{i} \leq x_{i}^{u} &
\end{array}
$$

Here, the design variables are thickness and lamination parameters in the following form

$$
x=\left\{\begin{array}{llllllll}
t_{c} \mid & t_{f} & \xi_{c}^{A} \mid & \xi_{f}^{A} \mid & \xi_{f}^{B u} \mid & \xi_{c}^{D} \mid & \xi_{f}^{D}
\end{array}\right\}^{T}
$$

which defines the stiffness matrices,

$$
\begin{gathered}
A=t_{c}\left[\xi^{A}\right]_{c}\{U\}_{c}+t_{f}\left[\xi^{A}\right]_{f}\{U\}_{f} \\
D=\frac{t_{c}^{3}}{12}\left[\xi^{D}\right]_{c}\{U\}_{c}+\frac{t_{f}^{3}}{12}\left[\xi^{D}\right]_{f}\{U\}_{f}+\frac{t_{c} t_{f}}{4}\left(t_{f}\left[\xi^{B u}\right]_{f}+t_{c}\left[\xi^{A}\right]_{f}\right)\{U\}_{f}
\end{gathered}
$$

where the subscripts $c$ and $f$ correspond to the core and facesheet material-blocks respectively.

Admissibility constraints for lamination parameters including those of $B_{l}$ and $B_{u}$ take familiar form as written by Kameyama and Fukunaga [21]:

$$
\text { Bounds: }-1 \leq \xi_{[1,2,3,4]}^{[A, B l, B u, D]} \leq 1
$$

Feasibility: For $k=A, B_{l}, B_{u}, D$,

$$
\begin{aligned}
& 2\left(\xi_{1}^{k}\right)^{2}-1 \leq \xi_{2}^{k} \leq 1-2\left(\xi_{3}^{k}\right)^{2}, \\
& 2\left(\xi_{2}^{k}+1\right)\left(\xi_{3}^{k}\right)^{2}-4 \xi_{1}^{k} \xi_{3}^{k} \xi_{4}^{k}+\left(\xi_{4}^{k}\right)^{2} \leq\left[\xi_{2}^{k}-2\left(\xi_{1}^{k}\right)^{2}+1\right]\left(1-\xi_{2}^{k}\right)
\end{aligned}
$$

Standard Angles: $\xi_{4}^{[A, B l, B u, D]}=0$ 

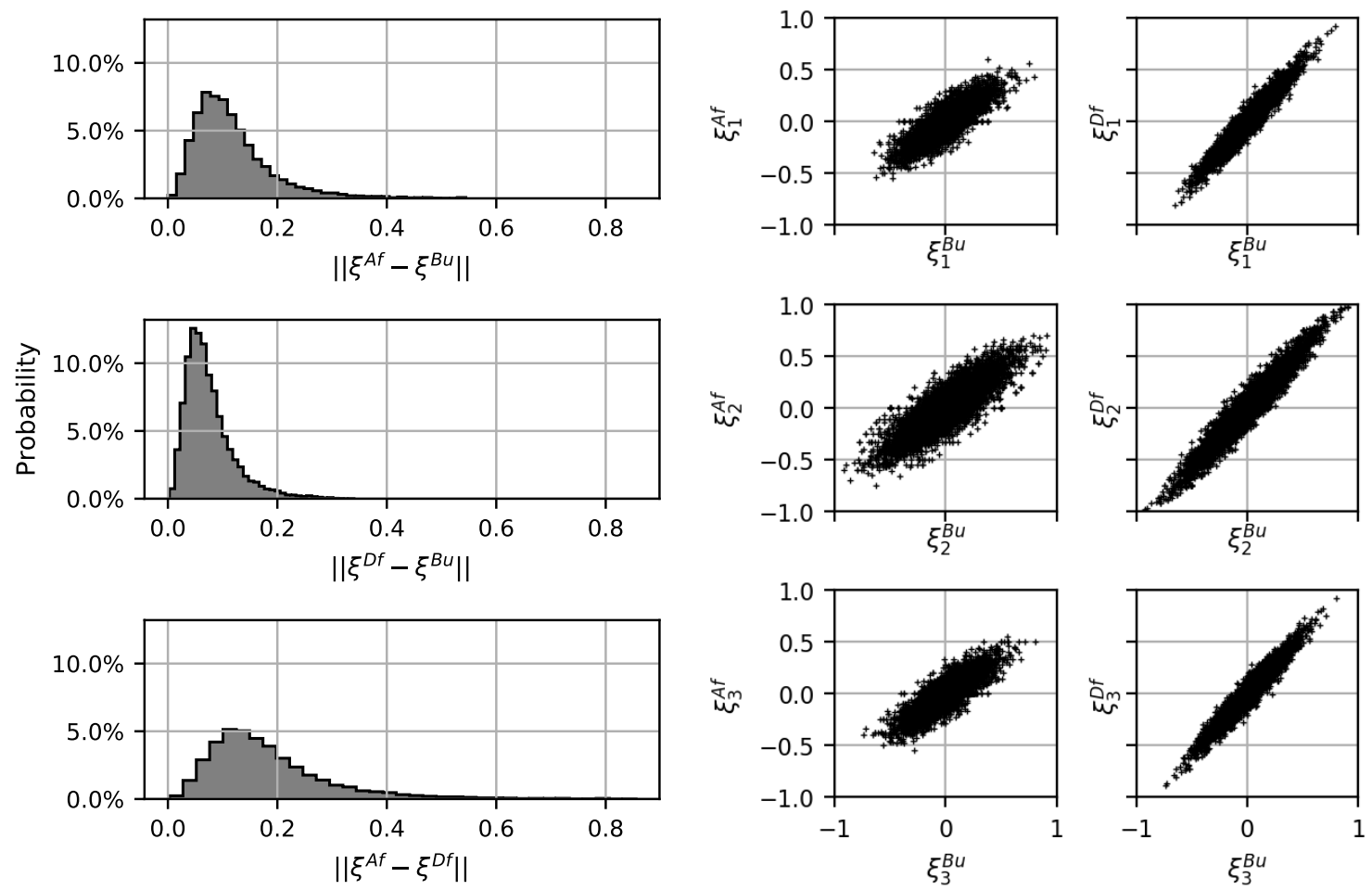

(a)

(b)

Fig. 6 Correlation between lamination parameters

Figure 7 contains the design curves resulting from this optimization problem. The problem was solved several times, once for each constant value of facesheet thickness. The curves include a comparison of the load-carrying ability of a standard laminate of the same thickness. Loads on the standard laminate were obtained by setting $t_{c}=0$ and neglecting the crimping failure mode. The shear stiffness of the core was also varied to see its effect on the crimping load. The nominal shear stiffness of the core and fictitiously improved shear stiffness by a factor of 2 and 3 are considered. The design curves are also generated using a Monte-Carlo simulation as shown in Figure 7. The similarity between the curves shows the ability of the developed parametrization scheme within an optimization environment.

Observing the main features of the curves reveal the following general characteristics for the case with the nominal core shear stiffness. Adding a core to thin facesheet panels enables a sandwich panel to carry significantly more load than the standard laminates. For thicknesses of up to 20 layers, the standard laminate fails by buckling, while facesheet failure at $\mathrm{OHC}$ is expected for the sandwich. This is observed in Figure 8 showing the excess loads for each failure mode at the optimal design. A value of 0 for excess loads implies that the particular failure mode is the design driver at that point.

Beyond 20 plies the sandwich fails by crimping. For loads of $2000 \mathrm{kN}$, the curves could suggest the designer should probably select a core material with a higher shear stiffness. This isn't always simple since core materials with higher shear stiffness are often more expensive and more difficult to manufacture. Additionally, the core shear stiffness of the panel maybe need to be knocked down to allow for impact damage tolerance. Choices such as aluminum cores will also mean the sandwich will become susceptible to factors such as rust. If no other core material is available sandwich structures should be avoided at this load level. This is because with a very shallow load carrying slope, a small increase in load requirements can require a very large increase in facesheet thickness (up to 3 times) or sudden catastrophic failure. The study demonstrated here assumes a maximum core thickness of $50 \mathrm{~mm}$. With both crimping and buckling improving as the core thickness is increased, it can be expected that having a larger core limit would result in the sandwich design being able to carry higher loads, limited then by OHC. This is also seen in Figure 9, showing the optimal core thickness with respect to the maximum allowed core thickness, for different facesheet thickness.

Beyond a total facesheet thickness of around 100 plies, the thickness of the standard laminate is sufficient to 


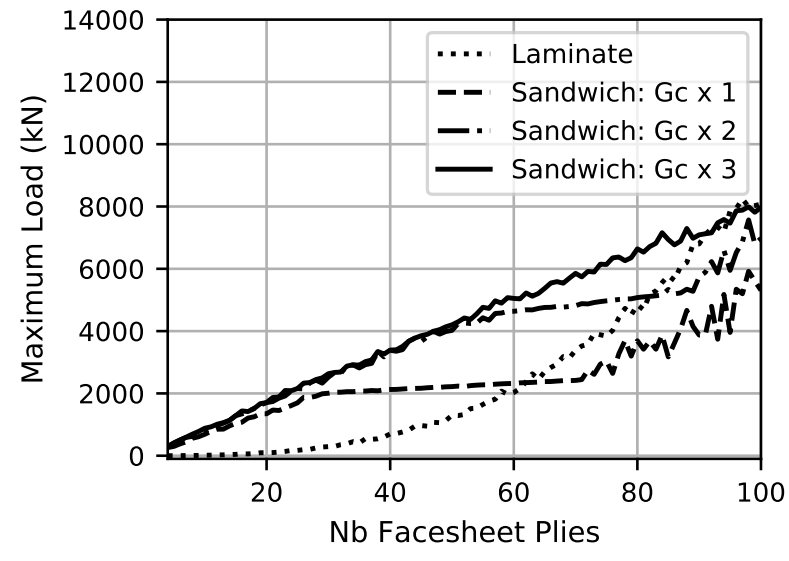

(a) Monte Carlo Simulation

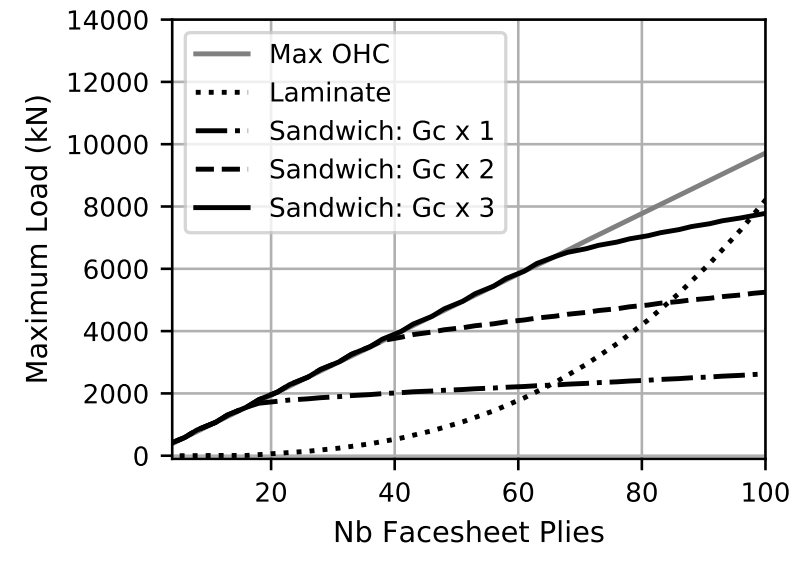

(b) Continuous Optimization

Fig. 7 Load-carrying ability for standard laminates and sandwich composites (sandwich core shear stiffness nominal, multiplied by factor 2, multiplied by factor 3)
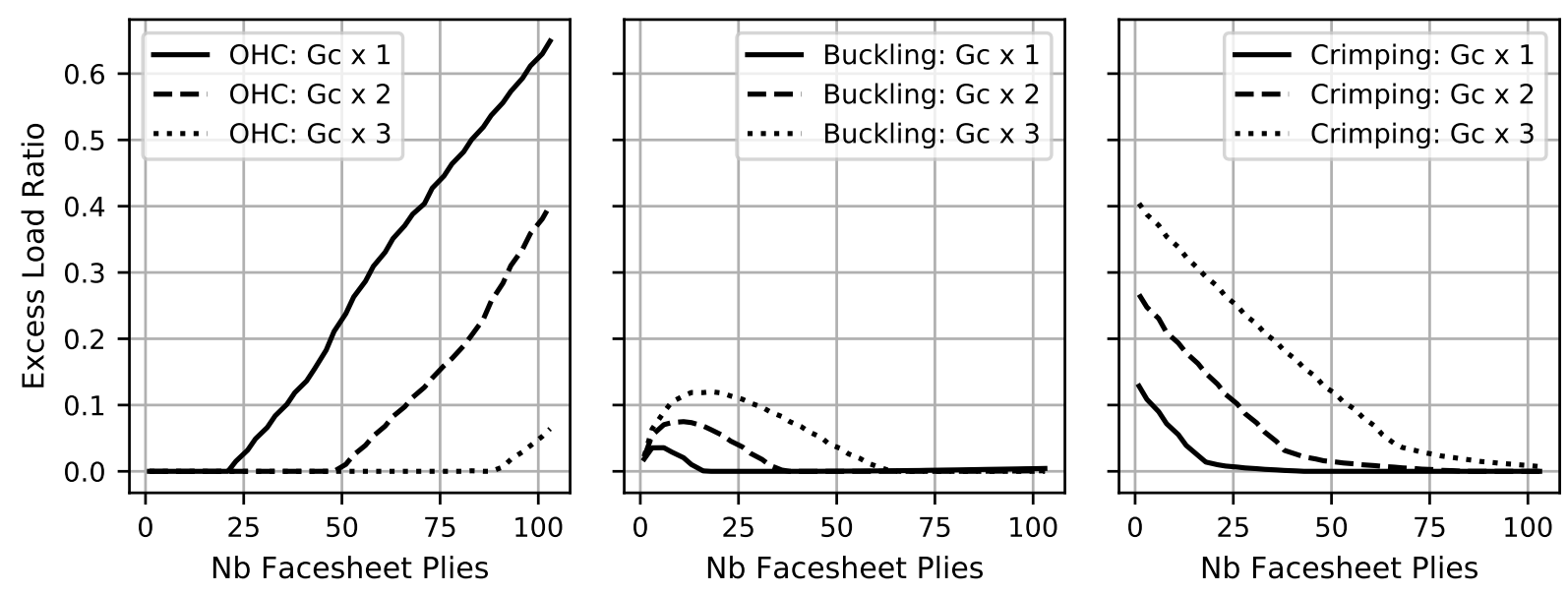

Fig. 8 Excess loads for each failure mode at the optimum design

withstand buckling loads, while the buckling load of a sandwich is reduced by core shear thickness. Beyond these load levels, standard laminates perform better. Figure 10 shows the lamination parameters for the optimum sandwich design obtained for each of the pre-set facesheet thicknesses for the sake of completeness.

\section{Conclusion}

This paper has presented a lamination parameters scheme to be used for sandwich panel design, including that of hybrid material. The parametrization scheme is analogous to classic lamination parameters; which allows a number of already available lamination parameter constraints to be applied to the newly proposed set without modification of form. Unlike previously-applied methods, the scheme developed includes parameters which provide an exact representation of the bending stiffness of offset material blocks; the omission of which, as was demonstrated, can lead to significant errors in important analysis results such as panel buckling. For applications where the user is willing to trade some accuracy for a reduction in computational cost, the paper also developed an improved approximation scheme, where no additional parameters need to be introduced. The efficacy of the parametrization scheme was demonstrated in a sample application to sandwich panel design. 


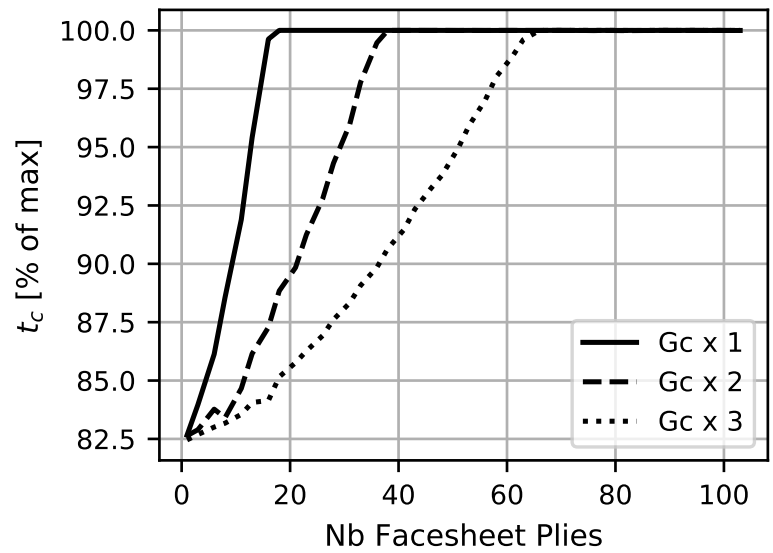

Fig. 9 Optimum core thickness

\section{References}

[1] Tsai, S. W., and Pagano, N. J., "Invariant properties of composite materials.” Tech. rep., Air Force Materials Lab WrightPatternson AFB Ohio, 1968.

[2] Hahn, H. T., and Tsai, S. W., Introduction to composite materials, CRC Press, 1980.

[3] Diaconu, C. G., Sato, M., and Sekine, H., "Feasible region in general design space of lamination parameters for laminated composites," AIAA Journal, Vol. 40, No. 3, 2002, pp. 559-565.

[4] Bloomfield, M., Diaconu, C., and Weaver, P., "On feasible regions of lamination parameters for lay-up optimization of laminated composites," Proceedings of the Royal Society of London A: Mathematical, Physical and Engineering Sciences, The Royal Society, 2009, pp. 1123-1143.

[5] Setoodeh, S., Abdalla, M., and Gurdal, Z., "Approximate feasible regions for lamination parameters," 11th AIAA/ISSMO Multidisciplinary Analysis and Optimization Conference, 2006, p. 6973.

[6] Miki, M., and Sugiyamat, Y., "Optimum design of laminated composite plates using lamination parameters," AIAA Journal, Vol. 31, No. 5, 1993, pp. 921-922.

[7] Yamazaki, K., "Two-level optimization technique of composite laminate panels by genetic algorithms," 37th Structure, Structural Dynamics and Materials Conference, 1996, p. 1539.

[8] Fukunaga, H., and Vanderplaats, G. N., "Stiffness optimization of orthotropic laminated composites using lamination parameters," AIAA Journal, Vol. 29, No. 4, 1991, pp. 641-646.

[9] Ijsselmuiden, S. T., Abdalla, M. M., and Gürdal, Z., "Optimization of variable-stiffness panels for maximum buckling load using lamination parameters," AIAA Journal, Vol. 48, No. 1, 2010, pp. 134-143.

[10] Liu, D., Toropov, V., Zhou, M., Barton, D., and Querin, O., "Optimization of Blended Composite Wing Panels Using Smeared Stiffness Technique and Lamination Parameters," 51st AIAA/ASME/ASCE/AHS/ASC Structures, Structural Dynamics, and Materials Conference, American Institute of Aeronautics and Astronautics, Orlando, Florida, 2010. doi:10.2514/6.2010-3079, URL http://arc . aiaa.org/doi/abs/10.2514/6.2010-3079.

[11] Dillinger, J. K. S., Klimmek, T., Abdalla, M. M., and Gürdal, Z., "Stiffness Optimization of Composite Wings with Aeroelastic Constraints," Journal of Aircraft, Vol. 50, No. 4, 2013, pp. 1159-1168. doi:10.2514/1.C032084, URL http: //arc.aiaa.org/doi/10.2514/1.c032084.

[12] Silva, G. H. C., do Prado, A. P., Cabral, P. H., De Breuker, R., and Dillinger, J. K. S., "Tailoring of a Composite Regional Jet Wing Using the Slice and Swap Method," Journal of Aircraft, Vol. 56, No. 3, 2019, pp. 990-1004. doi:10.2514/1.C035094, URL https: //arc .aiaa.org/doi/10.2514/1.C035094.

[13] Miki, M., and Sugiyama, Y., "Optimum design of laminated composite plates using lamination parameters," 32nd Structures, Structural Dynamics, and Materials Conference, American Institute of Aeronautics and Astronautics, Baltimore,MD,U.S.A., 1991. doi:10.2514/6.1991-971, URL http://arc . aiaa.org/doi/10.2514/6.1991-971. 

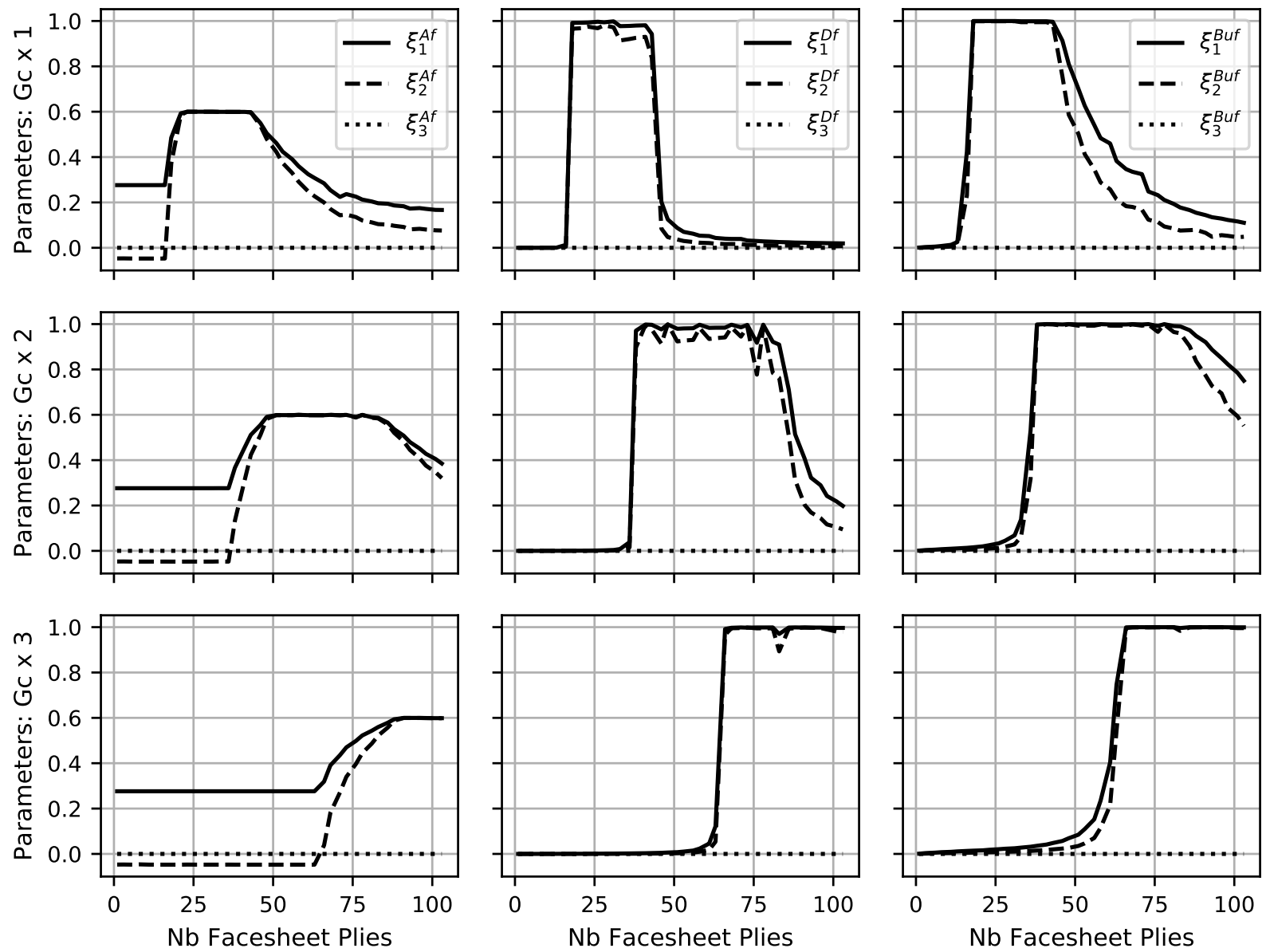

Fig. 10 Optimum lamination parameters

[14] Moh, J.-S., and Hwu, C., “Optimization for buckling of composite sandwich plates,” AIAA Journal, Vol. 35, No. 5, 1997, pp. $863-868$.

[15] Lin, C.-C., and Lee, Y.-J., "Stacking sequence optimization of laminated composite structures using genetic algorithm with local improvement," Composite structures, Vol. 63, No. 3-4, 2004, pp. 339-345.

[16] Yuan, C., Bergsma, O., Koussios, S., Zu, L., and Beukers, A., "Optimization of Sandwich Composites Fuselages Under Flight Loads," Applied Composite Materials, Vol. 19, No. 1, 2012, pp. 47-64. doi:10.1007/s10443-010-9180-9, URL http: //link. springer. com/10.1007/s10443-010-9180-9.

[17] Balabanov, V., Weckner, O., Epton, M., Mabson, G., Cregger, S., and Blom, A., "Optimal design of a composite sandwich structure using lamination parameters," 53rd AIAA/ASME/ASCE/AHS/ASC Structures, Structural Dynamics and Materials Conference, 20th AIAA/ASME/AHS Adaptive Structures Conference 14th AIAA, 2012, p. 1520.

[18] Weckner, O., and Balabanov, V., "Efficient design of shear-deformable hybrid composite structures," , Feb. 4 2014. US Patent $8,645,110$.

[19] Jin, P., Song, B., Zhong, X., Yu, T., and Xu, F., "Aeroelastic tailoring of composite sandwich panel with lamination parameters," Proceedings of the Institution of Mechanical Engineers, Part G: Journal of Aerospace Engineering, Vol. 230, No. 1, 2016, pp. 105-117. doi:10.1177/0954410015587724, URL http: // journals. sagepub. com/doi/10.1177/0954410015587724.

[20] Montemurro, M., Catapano, A., and Doroszewski, D., "A multi-scale approach for the simultaneous shape and material optimisation of sandwich panels with cellular core," Composites Part B: Engineering, Vol. 91, 2016 , pp. 458 - 472. doi:https://doi.org/10.1016/j.compositesb.2016.01.030, URL http://www . sciencedirect.com/science/article/pii/ S1359836816000652. 
[21] Kameyama, M., and Fukunaga, H., "Optimum design of composite plate wings for aeroelastic characteristics using lamination parameters," Computers \& Structures, Vol. 85, No. 3-4, 2007, pp. 213-224. doi:10.1016/j.compstruc.2006.08.051.

[22] Dillinger, J., Klimmek, T., Abdalla, M., and Gürdal, Z., "Stiffness Optimization of Composite Wings with Aeroelastic Constraints," Journal of Aircraft, Vol. 50, No. 4, 2013, pp. 1159-1168. URL https://elib.dlr . de/84219/.

[23] Dillinger, J., Cabral, P., Silva, G., Prado, A., and Meddaikar, M. Y., "CFD corrected static aeroelastic stiffness optimization of a passenger aircraft wing," AASST 2018 - 1st International Conference on Advances in Aerospace Structures, Systems \& Technology, 2018. URL https://elib.dlr.de/122040/.

[24] Fogarty, J. H., "Honeycomb core and the myths of moisture ingression," Applied Composite Materials, Vol. 17, No. 3, 2010, pp. 293-307.

[25] Herrmann, A. S., Zahlen, P. C., and Zuardy, I., "Sandwich Structures Technology in Commercial Aviation," Sandwich Structures 7: Advancing with Sandwich Structures and Materials, edited by O. Thomsen, E. Bozhevolnaya, and A. Lyckegaard, Springer Netherlands, Dordrecht, 2005, pp. 13-26.

[26] Feraboli, P., "Composite Materials Strength Determination Within the Current Certification Methodology for Aircraft Structures," Journal of Aircraft, Vol. 46, No. 4, 2009, pp. 1365-1374. doi:10.2514/1.41286, URL http://dx.doi .org/10.2514/1. 41286.

[27] Marlett, K., "Hexcel 8552 IM7 Unidirectional Prepreg 190 gsm \& 35\%RC Qualification Material Property Data Report," Tech. Rep. CAM-RP-2009-015 Rev A, National Institute for Aviation Research, Wichita, KS, Apr. 2011.

[28] "Design and Analysis of Sandwich Structures," Composite Materials Handbook - 17, Vol. 6, SAE International, Wichita, Kansas, 2017, Chap. 4, pp. 1-228.

[29] Reddy, J. N., Mechanics of Laminated Composite Plates and Shells: Theory and Analysis, CRC Press, 2003.

[30] Kassapoglou, C., "Sandwich Structure,” Design and Analysis of Composite Structures, John Wiley \& Sons Ltd, Oxford, UK, 2013, Chap. 10, pp. 275-307. doi:10.1002/9781118536933, URL http: //dx . doi . org/10.1002/9781118536933 . ch10. 